\title{
Requirements for resuming translation in chimeric transfer-messenger RNAs of Escherichia coli and Mycobacterium tuberculosis
}

\author{
Iwona K Wower ${ }^{1}$, Christian Zwieb ${ }^{2}$ and Jacek Wower ${ }^{1 *}$
}

\begin{abstract}
Background: Trans-translation is catalyzed by ribonucleprotein complexes composed of SmpB protein and transfer-messenger RNA. They release stalled ribosomes from truncated mRNAs and tag defective proteins for proteolytic degradation. Comparative sequence analysis of bacterial tmRNAs provides considerable insights into their secondary structures in which a tRNA-like domain and an mRNA-like region are connected by a variable number of pseudoknots. Progress toward understanding the molecular mechanism of trans-translation is hampered by our limited knowledge about the structure of tmRNA:SmpB complexes.
\end{abstract}

Results: Complexes consisting of M. tuberculosis tmRNA and E. coli SmpB tag truncated proteins poorly in E. coli. In contrast, the tagging activity of E. coli tmRNA is well supported by M. tuberculosis SmpB that is expressed in E. coli. To investigate this incompatibility, we constructed 12 chimeric tmRNA molecules composed of structural features derived from both E. coli and M. tuberculosis. Our studies demonstrate that replacing the hp5-pk2-pk3-pk4 segment of $E$. coli tmRNA with the equivalent segment of $M$. tuberculosis tmRNA has no significant effect on the tagging efficiency of chimeric tmRNAs in the presence of E. coli SmpB. Replacing either helices $2 \mathrm{~b}-2 \mathrm{~d}$, the single-stranded part of the ORF, pk1, or residues 79-89 of E. coli tmRNA with the equivalent features of M. tuberculosis tmRNA yields chimeric tmRNAs that are tagged at 68 to 88 percent of what is observed with $E$. coli tmRNA. Exchanging segments composed of either pk1 and the single-stranded segment upstream of the ORF or helices $2 \mathrm{~b}-2 \mathrm{~d}$ and pk1 results in markedly impaired tagging activity.

Conclusion: Our observations demonstrate the existence of functionally important but as yet uncharacterized structural constraints in the segment of tmRNA that connects its TLD to the ORF used for resuming translation. As trans-translation is important for the survival of $M$. tuberculosis, our work provides a new target for pharmacological intervention against multidrug-resistant tuberculosis.

Keywords: Chimeric tmRNA, SmpB, Trans-translation, Protein tagging

\section{Background}

Translation of mRNAs that are missing stop codons stalls ribosomes and produces truncated proteins. To recycle stalled ribosomes and degrade defective proteins, bacteria use trans-translation, a quality control process mediated by a ribonucleoprotein particle composed of transfer-messenger RNA (tmRNA) and SmpB $[1,2]$. The tmRNA acts as both tRNA and mRNA through its tRNA-like domain (TLD) and a mRNA-like region

\footnotetext{
* Correspondence: wowerja@auburn.edu

'Department of Animal Sciences, Auburn University, Auburn, AL 36849, USA

Full list of author information is available at the end of the article
}

(MLR) with an open reading frame (ORF) that encodes a short proteolysis-inducing tag peptide [3-5]. In the majority of bacterial species, the TLD and MLR segments are connected by four pseudoknots (pk1-pk4) (Figure 1). TmRNA binding to stalled ribosomes is facilitated by elongation factor $\mathrm{Tu}(\mathrm{EF}-\mathrm{Tu}), \mathrm{SmpB}$ and ribosomal protein S1. The SmpB molecule mimics the D and anticodon arms that are absent in tmRNA [6]. EF-Tu binds to the T-arm of the TLD precisely as observed in canonical aminoacyl-tRNAs [7,8]. Protein S1 was found in E. coli tmRNA:ribosome complexes that were assembled in vivo [9]. S1 has been shown to bind to free tmRNAs and tmRNA:SmpB complexes by contacting 


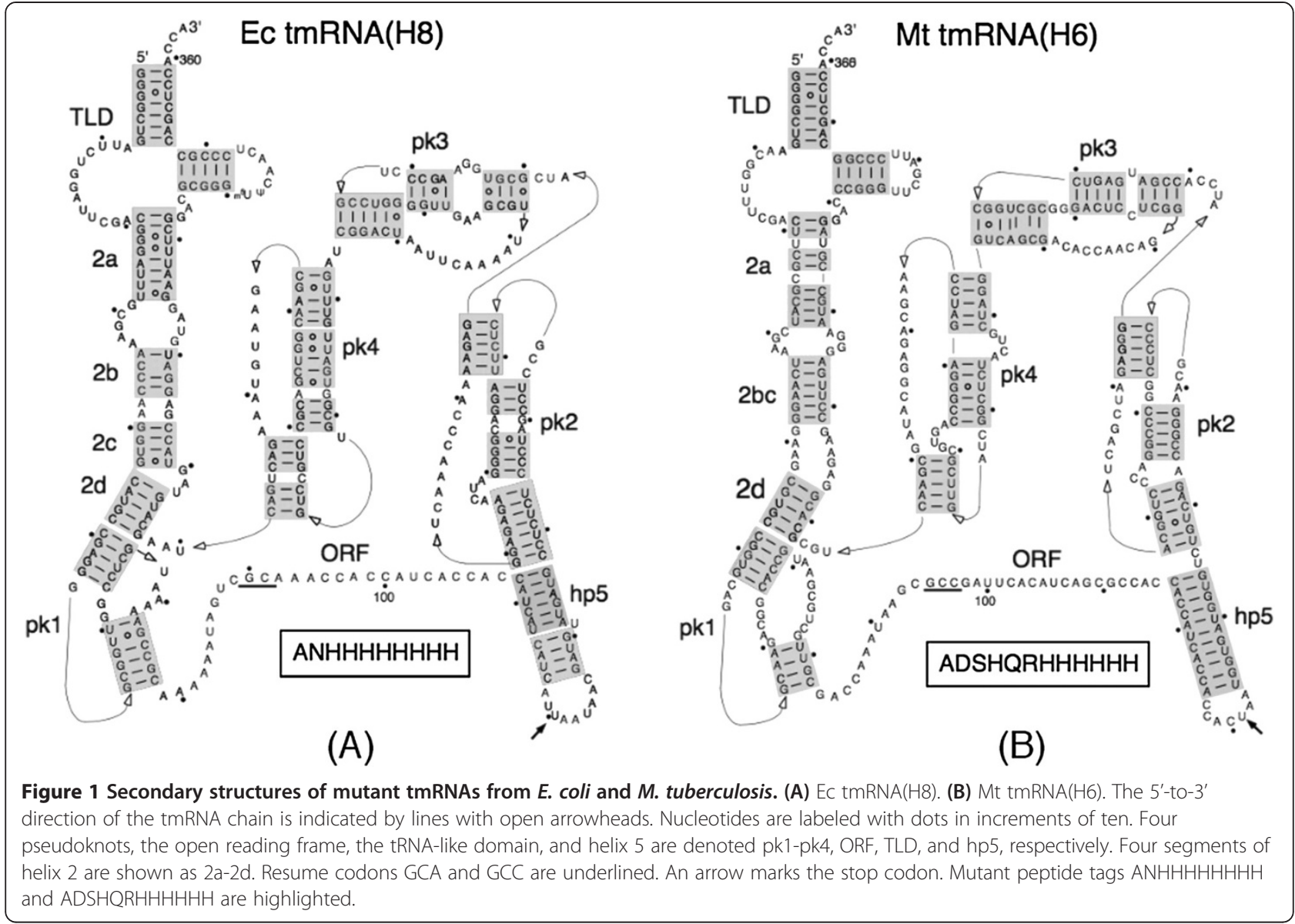

the MLR, pk2 and pk3 [10]. Although essential for trans-translation in M. tuberculosis, protein S1's contributions to trans-translation in E. coli and other bacteria remain poorly understood [11-14].

Cryo-electron microscopy (cryo-EM) studies yielded a number of snapshots of the tmRNA:SmpB complex as it binds to the A site of the stalled ribosome and is accommodated in the P site [15-20]. The TLD interacts with the ribosome similarly to a canonical tRNA whereas pk2-pk4 form an arc around the head of the $30 \mathrm{~S}$ subunit. While tmRNA maintains its overall structure during translocation from the A to the $\mathrm{P}$ site, the region encompassing helices $2 \mathrm{a}-2 \mathrm{~d}(\mathrm{hp} 2 \mathrm{a}-2 \mathrm{~d})$ and $\mathrm{pk} 1$ undergoes significant conformational changes. Molecular modeling suggests that pk1 unfolds, at least partially, as the TLD is translocated to the E site [21]. This suggestion is consistent with our earlier studies demonstrating that pk1 could be reduced to a hairpin without affecting tmRNA's tagging activity [22]. Although the resolution of cryo-EM images is not sufficiently high for observing subtle changes in the single stranded regions of the ribosome-bound tmRNA molecule, site-directed mutagenesis experiments revealed that the conserved nucleotides (85-UAG-86) located upstream of the ORF together with
SmpB are instrumental for setting the correct reading frame on E. coli tmRNA [23]. According to more recent cryo-EM studies, SmpB contacts at least five nucleotides upstream of the resume codon [18].

In this article, we explore structure-function relationships in tmRNA:SmpB complexes using chimeric tmRNA molecules that have been constructed by swapping equivalent segments of E. coli and M. tuberculosis tmRNAs. Although these tmRNAs have very similar predicted secondary structures, $E$. coli $\mathrm{SmpB}$ very poorly supports the tagging activity of $M$. tuberculosis tmRNA on E. coli ribosomes. In contrast, M. tuberculosis SmpB promotes the tagging activity of $E$. coli tmRNA to nearly the same level as the E. coli SmpB. These findings provided an opportunity to systematically scan the tagging activity the tmRNA with the aim to identify incompatibilities with the tagging activity of the heterologous M. tuberculosis tmRNA: E. coli SmpB complex. Our studies demonstrate that replacing the hp5-pk2-pk3-pk4 segment of $E$. coli tmRNA with the equivalent segment of M. tuberculosis tmRNA produces a chimeric molecule that efficiently tags truncated proteins in the presence of E. coli SmpB. Swapping helices $2 \mathrm{~b}-2 \mathrm{~d}$, pk1 and the singlestranded segment upstream of the ORF separately yields chimeric tmRNA:E. coli SmpB complexes with a decreased 
tagging activity. In contrast, replacing $E$. coli tmRNA segments composed either of helices $2 \mathrm{~b}-2 \mathrm{~d}$ and $\mathrm{pk} 1$ or of $\mathrm{pk} 1$ and single-stranded sequence upstream of the ORF with equivalent segments of $M$. tuberculosis tmRNA yields chimeric tmRNAs that tag very poorly in the presence of E. coli SmpB. Since SmpB does not interact with helices $2 \mathrm{~b}-2 \mathrm{~d}$ and $\mathrm{pk} 1$, our findings suggest the existence of an as yet uncharacterized tmRNA feature that plays an important role in trans-translation.

\section{Results}

In vivo tagging by $E$. coli and $M$. tuberculosis tmRNAs

SmpB protein binds to the TLD to form a complex that mimics the shape of canonical tRNA [6]. Cryo-EM studies of the E. coli TLD:SmpB complex accommodated in the $\mathrm{P}$ site suggest that conserved residues 134-140 (and possibly 18-24) of $\mathrm{SmpB}$ are in close proximity to five conserved tmRNA residues (positions 85-89) [18]. Given that the ORF and the pseudoknots of tmRNA can be extensively modified without affecting trans-translation $[24,25]$, one would expect that tmRNA molecules of similar size and secondary structure, like the canonical tRNAs, are active in heterologous transtranslational systems. To test this conjecture, we used tmRNA(H8), a fully functional derivative of $E$. coli tmRNA in which the ORF has been modified to contain eight histidine codons (Figure 1A). In vivo, tmRNA(H8) tags truncated ribosomal protein L27 with a histidine-rich polypeptide $\left(\mathrm{ANH}_{8}\right)$ to yield a fusion protein that is resistant to proteolysis and therefore can be easily detected in fractionated $E$. coli lysates by staining with Coomassie Blue or by Western blot analysis [24]. To investigate the tagging activities of the $M$. tuberculosis tmRNA, a similar construct, Mt tmRNA(H6), that encodes the protease resistant $\mathrm{ADSHQRH} \mathrm{H}_{6}$ tag was synthesized (Figure 1B). The plasmid-encoded $\mathrm{Mt} \operatorname{tmRNA}(\mathrm{H} 6)$ was expressed in E. coli cells as efficiently as Ec tmRNA(H8) [24].

The main components of our in vivo tagging system are six variants of plasmid pWOW (Additional file 1: Figure S1 and Table S1) that express tmRNA-directed His-tagged truncated L27 in the presence of protein SmpB. Consistent with our previous studies, the plasmid-encoded E. coli tmRNA(H8) and SmpB efficiently tagged protein L27 in IW764, an E. coli strain lacking both the $s s r A$ gene (which encodes tmRNA) and the $\operatorname{smp} B$ gene (Figure 2, lane 2) [24]. In contrast, the tagging of truncated protein $L 27$ by M. tuberculosis tmRNA(H6) and E. coli SmpB was largely ineffective (Figure 2, lane 6). No tagging of truncated L27 was observed in E. coli IW764 that was transformed with pWOW derivatives encoding M. tuberculosis SmpB (Figure 2, lanes 3 and 5).

Further studies using Northern blotting revealed that the mRNAs for E. coli and M. tuberculosis SmpB were expressed well in E. coli IW764 cells (data not shown).

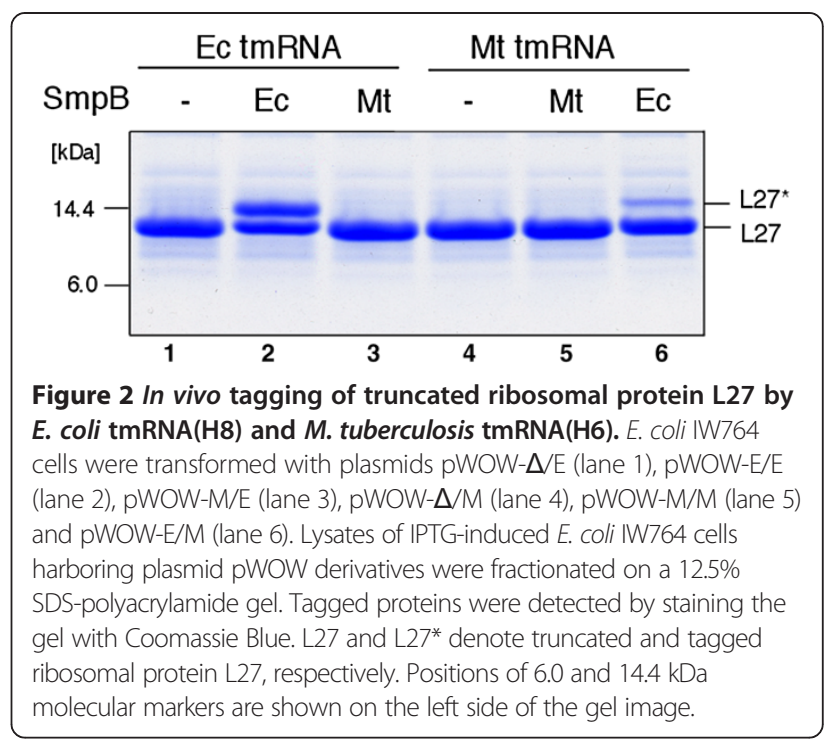

However, while E. coli $\mathrm{SmpB}$ protein was efficiently translated, no $M$. tuberculosis SmpB protein could be detected in cell lysates using Western blotting (Figure 3, lanes 3 and 6). This finding was unexpected because M. tuberculosis SmpB cloned in a plasmid vector under the control of the T7 promoter can be efficiently overexpressed in E.coli IW764 strain (see Methods). Translation of $M$. tuberculosis SmpB was likely repressed by inhibitory RNA structures encompassing the ribosome-binding site in its

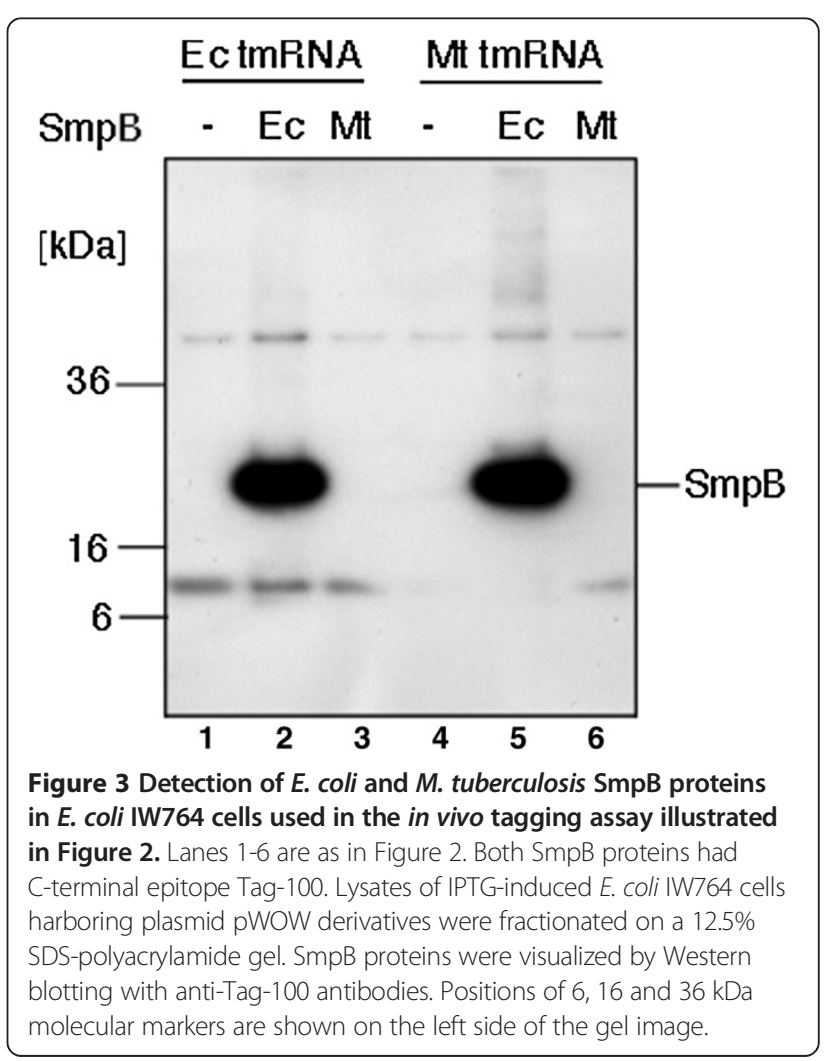


mRNA. Such structures were predicted to form only in $M$. tuberculosis SmpB mRNAs that were transcribed under control of its natural promoter [26].

\section{In vivo tagging activity of chimeric tmRNAs}

To identify regions of the $M$. tuberculosis tmRNA that impair its tagging activity in the presence of $E$. coli SmpB, we constructed 12 chimeric tmRNAs (E1-E9 in Figure 4; M1-M3 in Figure 5; see also Additional file 1: Table S2). All chimeras contained M. tuberculosis sequences within the hp5-pk2-pk3 segment known to have no role in the binding to $\mathrm{SmpB}$. In vivo tagging activities of chimeric tmRNA:E. coli SmpB complexes were analyzed by SDS-PAGE (Figure 6A). As E. coli tmRNA (H8) encodes a ten amino acid-long tag ANHHHHHHHH (Figure 1A), its activity produced a fast-migrating L27 protein derivative (Figure 6A, lane 1). M. tuberculosis tmRNA(H6) and chimeric tmRNA E1, both encoding a 12-mer ADSHQRHHHHHH (see Figure 1B), produced slower-migrating $\mathrm{L} 27$ protein derivatives that readily bind to Ni-NiTA magnetic agarose beads (see also Figure 7A).
Unexpectedly, well-tagging chimeric tmRNAs E2-E7, all of which encode a 12-mer ANDEHHНHНHНH, produced abnormally slow-migrating L27 derivatives. The abnormally slow mobility of the ANDEHHНHНHНHtagged L27 protein in a polyacrylamide gel can be explained by an enhanced tendency of protein segments containing histidyl residues to form a helical structure in the presence of SDS [27] and by an intimate link between increased SDS binding to helical structures [28].

Coomassie Blue stained gels were scanned, the tagging efficiencies of chimeric tmRNAs were measured and compared to the tagging efficiency of $E$. coli tmRNA (H8) as described in Methods (Figure 4). Figure 6A shows that replacing either pk1 or $\mathrm{hp} 2 \mathrm{~b}-2 \mathrm{~d}$ reduces the in vivo tagging activity of E6 and E7 by 12 to 14 percent. Replacing either the single-stranded portion of $E$. coli ORF (ssORF) or the single-stranded sequence upstream of the ORF with the equivalent $M$. tuberculosis segments yielded E3 (data not shown) and E5 that displayed approximately 70 percent tagging activity of E. coli tmRNA (H8) (see also Additional file 1:

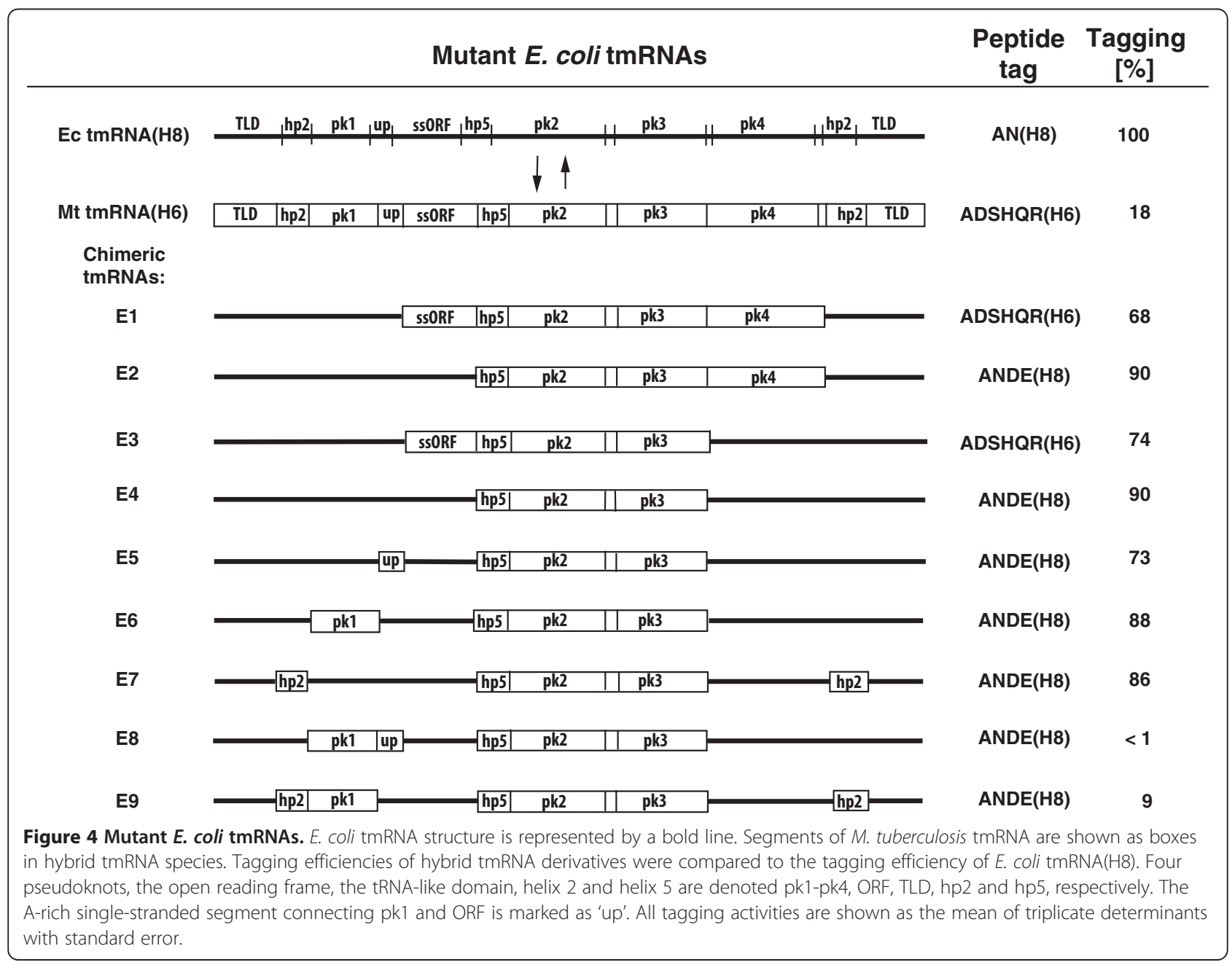



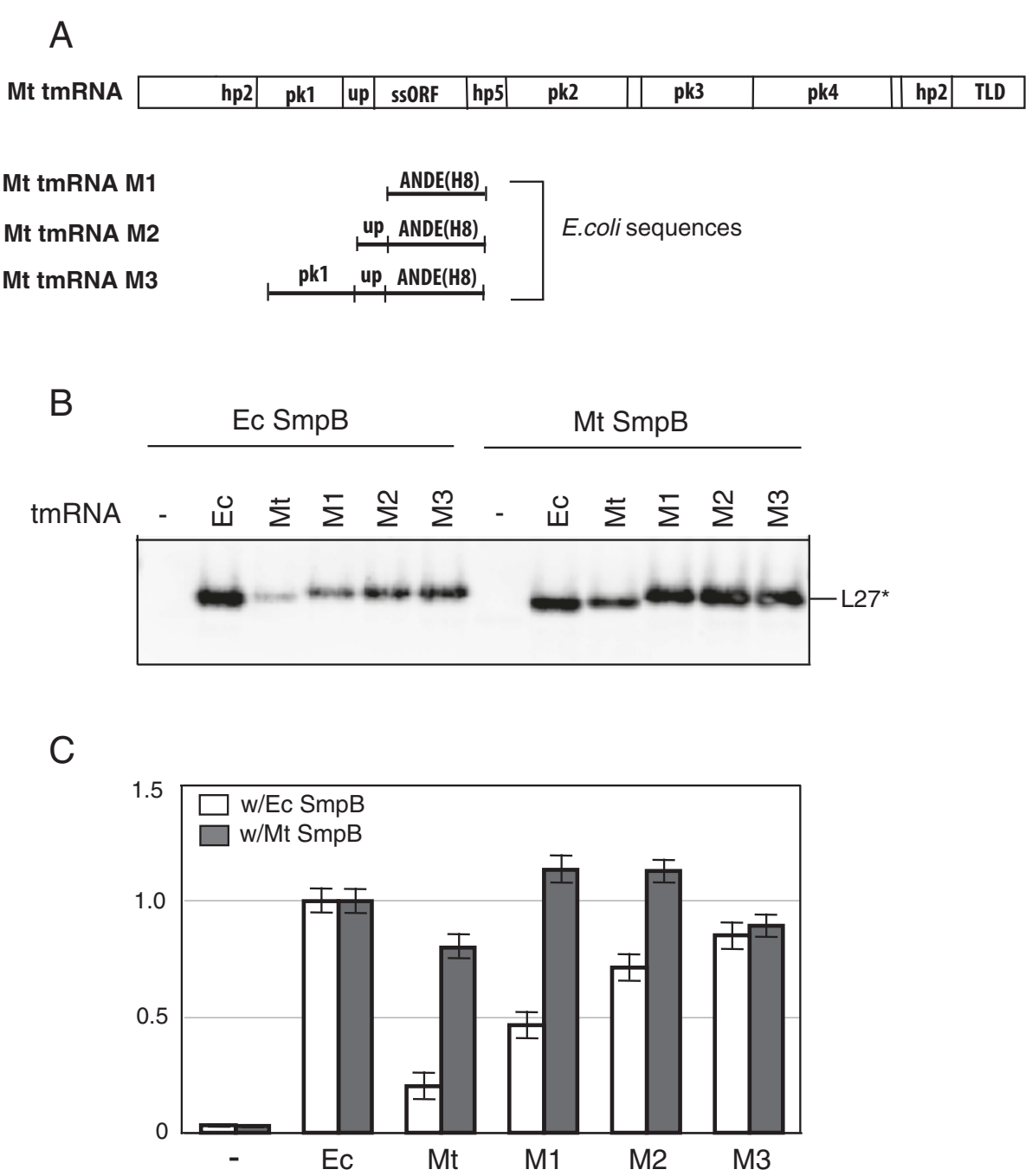

Figure 5 In vitro tagging of a truncated ribosomal protein L27 by mutant $M$. tuberculosis tmRNAs in the presence of $E$. coli and $M$. tuberculosis SmpB proteins. (A) Chimeric tmRNAs M1, M2 and M3 were created by replacing three segments of $M$. tuberculosis tmRNA with corresponding segments of E. coli tmRNA. Four pseudoknots, the single-stranded portion of the open reading frame, the tRNA-like domain, helix 2 and helix 5 are denoted pk1-pk4, ssORF, TLD, hp2 and hp5, respectively. The single-stranded sequence connecting pk1 and ORF is marked as 'up'. ORFs of E. coli and M. tuberculosis tmRNAs encode ANDE(H8) and ADSHQR(H6) peptide tags, respectively. (B) In vitro tagging of truncated ribosomal protein $\mathrm{L} 27$ by chimeric tmRNAs M1, M2 and M3 in the presence of either E. coli or M. tuberculosis SmpB proteins. Tagging was visualized by Western blotting with anti-T7 tag antibodies in ECL-Plex system. L27* denotes tagged ribosomal protein L27. (C) Graphical representation of Typhoon-derived data derived from four Western blotting analyses. Tagging efficiencies of hybrid tmRNA derivatives were normalized and compared to the tagging efficiency of the E. coli tmRNA(H8). Error bars show the standard deviation of three or more independent experiments.

Table S2). The most dramatic decrease of in vivo tagging was observed in E8 and E9 that have either M. tuberculosis hp2b-2d and pk1 or M. tuberculosis pk1 and singlestranded nucleotides upstream the ORF. This finding was unexpected because replacing individually $\mathrm{hp} 2 \mathrm{~b}-2 \mathrm{~d}$, pk1 and the single-stranded sequence upstream of the ORF had a relatively small effect on the tagging in vivo (Figure 6A, lanes E5-E7).

To determine whether the tagging inefficiencies of $M$. tuberculosis tmRNA(H6) and chimeric tmRNAs E8 and E9 are caused by their poor binding to stalled ribosomes, we measured the ribosomal occupancies of $E$. coli SmpB and tmRNA. Western blotting analysis demonstrated that the inefficient tagging of $M$. tuberculosis tmRNA(H6), and chimeric tmRNAs E8 and E9 was not caused by the inability of $E$. coli $\mathrm{SmpB}$ to associate with the ribosome (Figure 6B). Similar amounts of the ribosome-bound SmpB were found in the efficiently tagging E. coli tmRNA (H8) and the extremely poorly tagging chimeric tmRNA E8 preparations. Northern blot analysis of the ribosome-bound tmRNAs produced a difficult to interpret yet reproducible profile (Figure 6C). 


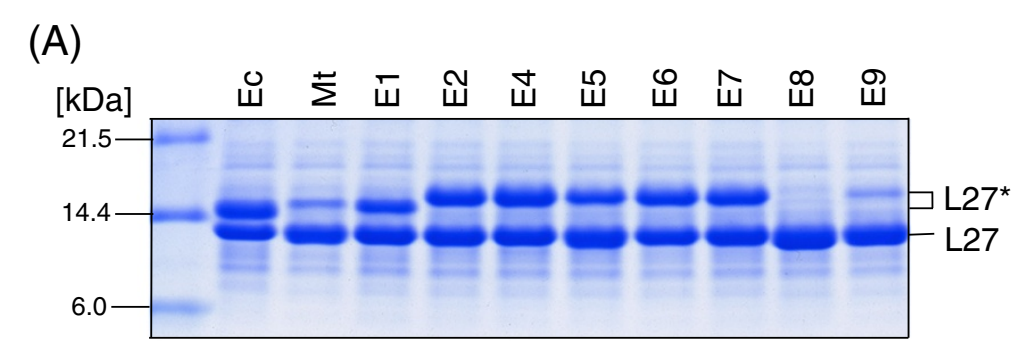

(B)

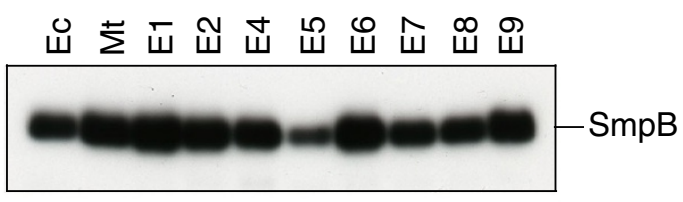

(C)

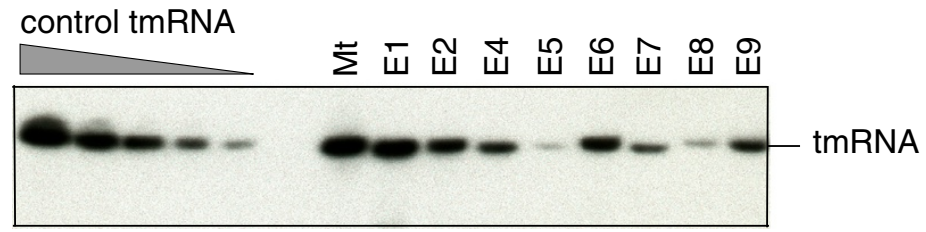

Figure 6 In vivo tagging of truncated ribosomal protein L27 by hybrid tmRNAs in the presence of E. coli SmpB protein. (A) Lysates of cells expressing E. coli (EC), M. tuberculosis (Mt) and hybrid tmRNAs (E1-E9) were fractionated on a 12.5\% SDS polyacrylamide gel. Truncated and tagged proteins were detected by staining the gel with Coomassie Blue. L27 and L27* denote truncated and tagged ribosomal protein L27, respectively. Molecular markers of 6.0, 14.4 and $21.5 \mathrm{kDa}$ are shown on the left side of the gel image. (B) Western analysis of the ribosome-bound E. coli SmpB. Aliquots of $1.0 \mathrm{~A}_{260}$ units of $70 \mathrm{~S}$ ribosomes derived from lysates of IPTG-induced E. coli IW764 cells harboring plasmid pWOW derivatives were fractionated on a 10\% SDS-polyacrylamide gel. The SmpB protein was visualized by Western blotting with polyclonal anti-E. coli SmpB antibodies. (C) Northern analysis of the ribosome-bound tmRNAs present in the tagging reaction mixture. Aliquots of $0.5 \mu \mathrm{g}$ RNA extracted from $70 \mathrm{~S}$ ribosomes were separated on a $6 \%$ denaturing polyacrylamide gel and blotted to a Zeta-probe membrane. [5'- $\left.{ }^{32} \mathrm{P}\right]$-labeled oligonucleotide probes complementary to a segment of M. tuberculosis tmRNA were hybridized to each tmRNA. To estimate quantity of tmRNA in the tmRNA:ribosome complexes, increasing amounts of purified $M$. tuberculosis tmRNA(H6) $(0.0125,0.025,0.05,0.1$ and 0.2 pmoles) were also fractionated.

Ribosomal occupancies of the poorly tagging $M$. tuberculosis tmRNA (H6) and the efficiently tagging chimeric tmRNA E1 were similarly pronounced while ribosomal occupancies of the poorly tagging chimeric tmRNA E8 and the well tagging E5 were similarly reduced. This may be explained by a scenario where the tmRNA E5 is either readily degraded or quickly cleared off the trans-translating ribosomes.

\section{In vitro tagging activity of chimeric tmRNAs}

In order to characterize the chimeric tmRNAs further, we analyzed their tagging properties in vitro. These assays were carried out in the $E$. coli $\mathrm{T} 7$ transcription/translation system for circular DNA (Promega) and employed the plasmid pETrpmA-At-1 that encodes truncated L27 [24]. Reaction mixtures were supplemented with purified transcripts of chimeric tmRNAs and purified SmpBs from either E. coli or M. tuberculosis.

The in vitro tagging pattern of chimeric tmRNA:E. coli $\mathrm{SmpB}$ complexes is shown in Figure 7A. As demonstrated in vivo, E8 and E9 tag truncated ribosomal protein L27 poorly ( $<1$ to 9 percent) at levels slightly higher than the degree of tagging observed with $M$. tuberculosis tmRNA (H6). This observation confirms that as yet uncharacterized structural features in the segment of $M$. tuberculosis tmRNA encompassing hp2b-2d, pk1 and the singlestranded sequence upstream of the ORF are responsible for the tagging inefficiency of $M$. tuberculosis tmRNA:E. coli SmpB complexes.

Figure 7A shows that the chimeric tmRNAs tag at different levels when analyzed in vitro in the presence of M. tuberculosis SmpB. Quantitative analysis of data derived from the in vitro experiments shows that $M$. tuberculosis $\mathrm{SmpB}$ is more effective in supporting the tagging activity of $E$. coli tmRNA(H8) than M. tuberculosis tmRNA(H6) (Figure 7B). Given that E5 is the least active chimeric tmRNA, the inefficient tagging of homologous M. tuberculosis tmRNA(H6):SmpB complexes on E. coli stalled ribosomes is likely caused by the single-stranded nucleotides upstream of the ORF. This observation is in conflict with an earlier study showing that replacing 85-UAGUC-89 with 85-UAAGC-89 in E. coli tmRNA does not affect the activity of the mutant tmRNA in the KanR assay [20]. 


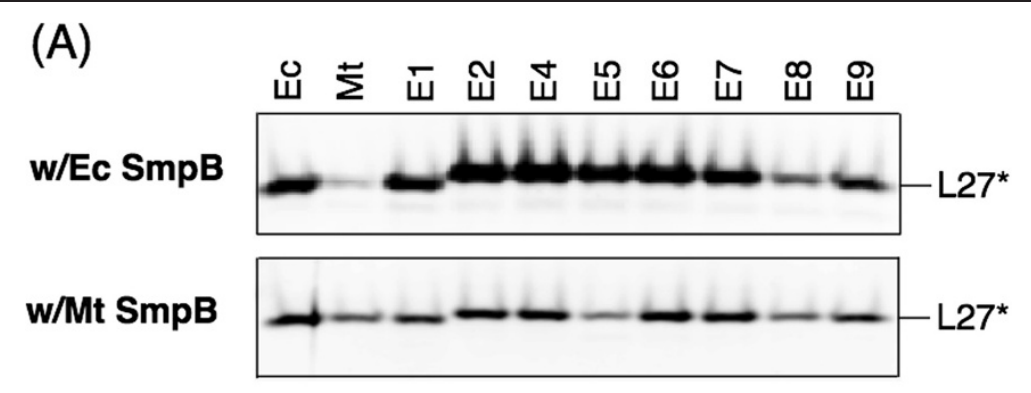

(B)

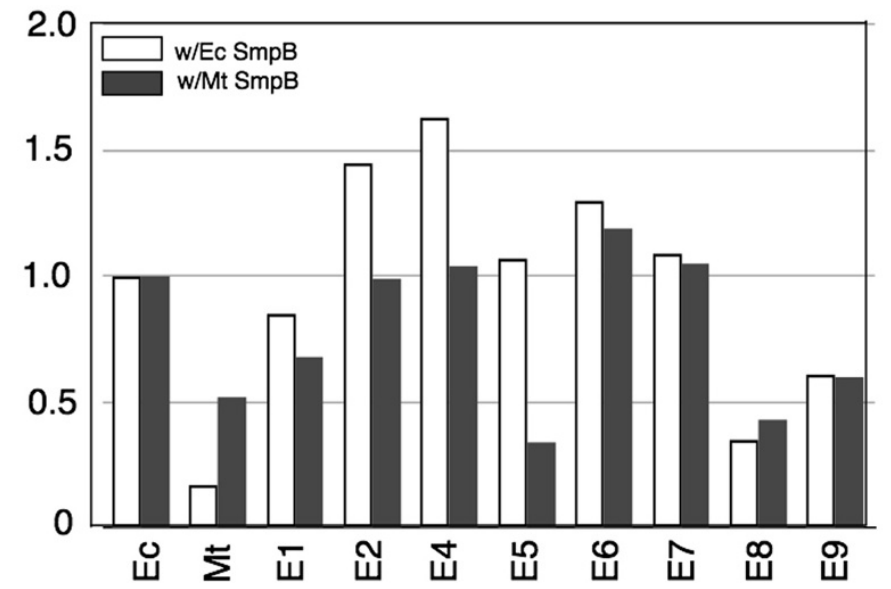

Figure 7 In vitro tagging of truncated ribosomal protein L27 by hybrid tmRNAs in the presence of either E. coli or M. tuberculosis SmpB proteins. (A) Western Blot analysis. Tagging reactions were assembled by addition of circular plasmid pETrpmA-At encoding truncated protein L27, purified SmpB proteins and tmRNAs to the T7 S30 transcription/translation mixture (Promega). After 60 min incubation at $37^{\circ} \mathrm{C}$, tagged proteins were captured on $\mathrm{Ni}^{2+}$-NTA-Sepharose and then fractionated on a 10\% SDS-polyacrylamide gel. Tagging was visualized by Western blotting with anti-T7 tag antibodies in ECL-Plex system. L27* denotes tagged ribosomal protein L27. (B) Graphical representation of Typhoon-derived data from the Western blot. Tagging efficiencies of hybrid tmRNA derivatives were normalized and compared to the tagging efficiency of the $E$. coli tmRNA(H8):SmpB complex. Error bars show the standard deviation of three or more independent experiments.

Tagging activity of $M$. tuberculosis tmRNAs derivatized with segments of $E$. coli tmRNA

As demonstrated above, replacing hp2b-2d, pk1, the single-stranded sequence upstream of the ORF and the ssORF in $E$. coli tmRNA with equivalent segments of $M$. tuberculosis tmRNA affects, to a variable extent, tagging activities of chimeric tmRNAs E3, E5, E6 and E7 (Figure 4). Results obtained using chimeric tmRNAs M1, M2 and M3, constructed by replacing pk1, the single-stranded sequence upstream of the ORF and the ssORF in $M$. tuberculosis tmRNA with equivalent segments of $E$. coli tmRNA, were equally revealing (Figure 5A). As seen in Figures $5 \mathrm{~B}$ and $5 \mathrm{C}$, replacing the ssORF in $M$. tuberculosis tmRNA with the equivalent segment of $E$. coli tmRNA had the most dramatic effect on tagging activities of the resulting chimeric tmRNAs M1, M2 and M3. When tested with E. coli SmpB, M1, M2 and M3 displayed respectively 2.4, 3.9 and 4.6-fold higher tagging activities than M. tuberculosis tmRNA(H6). In contrast, the chimeric tmRNA M1 was the most active when tagged in vitro in the presence of M. tuberculosis SmpB (Figures 5B and 5C). The gradual increase of the tagging activity in chimeric tmRNAs M1 and M2, and the inefficient tagging displayed by the chimeric tmRNA E8 derivative in the presence of E. coli SmpB suggest that the structural relationship between pk1 and the sequence upstream of the ORF is an important determinant for establishing proper interactions within the E. coli tmRNA:SmpB complex. Because the tag template after the +2 codon can be replaced by many different sequences without any loss of activity [24,29], it seems that the efficient tagging activity of the chimeric tmRNA M1: M. tuberculosis SmpB complex on E. coli ribosomes results from replacing the GCA-AAC sequence of the resume and +2 codon with the GCC-GAU sequence. Because changing the GCA triplet for the GCC triplet in the resume codon has very little effect on $E$. coli tmRNA tagging activity, the first nucleotide of the +2 codon is likely to enhance the tagging activity of chimeric tmRNA E1 in vitro [30]. This is 
consistent with a conservation of adenosine residues in the first two positions of the +2 codon [31].

\section{Discussion}

The ssrA gene is present in all known bacterial and some organellar genomes [32,33]. Its transcription produces single-chain tmRNA molecules in most bacteria and two-piece tmRNAs in certain major lineages that share similar architectures [34,35]. The most conserved features include the tRNA-like domain (TLD) and mRNA-like region (MLR) with an open reading frame (ORF). As shown in Figure 1, they are connected to each other by a number of short helices (hp2a-2d) and four pseudoknots (pk1-pk4). The TLD, hp2d, part of pk1, a number of nucleotides in the single-stranded sequence upstream of the ORF and the resume codon are highly conserved [36]. In contrast, the nucleotide composition and the length of the ORF can be highly variable. Because only the resume and +2 codons display biases for certain nucleotide triplets, a large portion of the ORF could be replaced by unrelated sequences without affecting the tagging activity of tmRNA [24,31]. Such conservation pattern suggests that tmRNAs, like canonical tRNAs, might be able to support protein synthesis in the distantly related bacteria. Results derived from our experiments with tmRNAs derived from E. coli (Proteobacteria) and M. tuberculosis (Actinobacteria) support this idea. Both tmRNA molecules tag, albeit with different efficiencies, truncated proteins in E. coli. M. tuberculosis SmpB effectively promotes tagging activities of both the $E$. coli and the M. tuberculosis tmRNAs. In contrast, E. coli SmpB supports the tagging activity of $M$. tuberculosis tmRNA very poorly. Our present studies demonstrate that replacing the hp5-pk2-pk3-pk4 segment of E. coli tmRNA with the equivalent segment from $M$. tuberculosis tmRNA have very little effect on the tagging activity of the resulting chimeric tmRNA E2 molecule. This is consistent with earlier studies, which demonstrated that extensive changes could be introduced into hp5 without significant effect on E. coli tmRNA tagging activity as long as the base pairing of hp5 is maintained [24]. While interchanging pk2, pk3 and pk4 causes only minor losses in tagging activity of mutant tmRNAs, disrupting the structure of these three pseudoknots has a differential effect not only on tmRNA tagging activity but also on its maturation $[24,25]$. Together, these findings indicate that the role of $\mathrm{pk} 2$, pk3 and $\mathrm{pk} 4$ is to maintain proper overall folding of the tmRNA molecule.

Replacing the ssORF in E. coli tmRNA with the equivalent segment of $M$. tuberculosis tmRNA reduces its tagging activity by about 30 percent. In contrast, replacing the ssORF in $M$. tuberculosis tmRNA with the equivalent segment of $E$. coli tmRNA increases its tagging activity to about 130 percent of the activity observed with an unmutated $M$. tuberculosis tmRNA. The sequence comparison of the ORF region in all known tmRNAs shows conservation only in the resume codon and the +2 codon [31]. Therefore, the increased tagging activity of the chimeric tmRNA M1, which uses GCA instead of GCC as its resume codon, can be explained by the differences in the availability of tRNA isoacceptors that decode these two codons. According to earlier studies, the GCA triplet is decoded by Ala-tRNA $1 \mathrm{~B}$ that belongs to the most abundant tRNAs in E. coli and constitute about five percent of the total tRNA. In contrast, the GCC triplet is decoded by the tRNA 2 isoacceptor that constitutes only about one percent of the total tRNA $[37,38]$.

The importance of the +2 codon for tmRNA functions is highlighted by studies of O'Connor [39] and Thibonnier et al. [40]. They demonstrated that translation of the resume codon alone is sufficient for both adding a minimal tag of two amino acids to a truncated protein and tmRNA-dependent ribosome recycling in $E$. coli, respectively. However, when two stop codons are introduced immediately downstream from the resume codon, the resulting mutant tmRNA is unable to complement the slow growth phenotype of $E$. coli lacking the chromosomal copy of the ssrA gene. When tagging activity of $M$. tuberculosis tmRNA is tested in E. coli, it is possible that the +2 codon (GAU) compensates a poor resume codon (GCC). This compensation relates most likely to the GAUdecoding tRNA Asp that is more abundant (3.72\%) than the AAC-decoding tRNA ${ }^{\text {Asn }}$ (1.85\%) [38]. However, additional studies are required to assess the contribution of the +2 codon to the tagging activity of the tmRNA molecule in general and to explain why adenosine is preferred at the second position of the +2 codon in particular [31].

The properties of pk1 and the single-stranded sequence immediately upstream the ORF have been studied extensively. Although residues 49-53 and 64-72 (E. coli tmRNA numbering; see Figure 1A) of pk1 are conserved [36], replacing pk1 with a single-stranded RNA yields a mutant tmRNA derivative that tags truncated proteins efficiently in vivo [22]. TmRNA sequence comparisons showed a strong preference for AUAG and AUAA tetramers upstream of the resume codon [41]. Mutations in these tetramers lead to either -1 or +1 frameshifting $[23,42,43]$. Given that both E. coli and M. tuberculosis tmRNAs exhibit the same sequence preference, a $27 \%$ drop in tagging activity of the chimeric tmRNA E5, which has $M$. tuberculosis single-stranded upstream sequence, was unexpected. Equally unexpected was either total or almost total tagging inactivity of $E$. coli tmRNA mutants having $M$. tuberculosis segments composed either of helices $2 \mathrm{~b}-2 \mathrm{~d}$ and $\mathrm{pk} 1$ or of pk1 and the single-stranded segment upstream of the ORF. Because sequences forming hp2b and hp2c are poorly conserved and because both $E$. coli and $M$. tuberculosis pk1 and single-stranded 
upstream sequence share almost all of the conserved nucleotide residues, the observed tagging defect must be induced by changes in the structure of the linker formed by hp2, pk1 and a single-stranded upstream sequence. Such changes are likely to affect the binding of SmpB protein to tmRNA. Two copies of this protein are able to bind to a single tmRNA molecule both on and off the ribosome $[16,44,45]$. One of them interacts with and cross-links to the $\mathrm{T}$ loop of the tmRNA [44]. This interaction is believed to play a minor, if any, role in tmRNA functions on stalled ribosomes. In contrast, the second SmpB molecule interacts with two segments of the ribosome-bound tmRNA. X-ray analysis revealed that it binds to the TLD to play a role of the missing $\mathrm{D}$ arm and its C-terminal part of beta 7 strand mimics the anticodon loop [6]. Moreover, the conserved residues of the beta 5 strand of the second $\mathrm{SmpB}$ molecule orient hp2A toward the decoding site of the $30 \mathrm{~S}$ ribosomal subunit. According to cryo-EM studies, when the TLD is accommodated in the $\mathrm{P}$ site, the 85-UAGUC-99 pentamer in the single-stranded upstream sequence is in proximity to and most likely interacts with the residues 134-140 in the C-terminus of E. coli SmpB [18]. As established by a number of earlier studies, this interaction is essential for a proper selection of the resume codon $[41,42,46]$. Our present work revealed that changes in the linker region are able to inactivate the tagging activity of tmRNA. Given that both $M$. tuberculosis and E. coli SmpB proteins support the tagging activity of $M$. tuberculosis tmRNA, one can speculate that the structure of its hp2-pk1-upstream sequence linker better accommodates the requirements of both SmpB proteins. Future studies will reveal in detail the structural features in the linker region that tmRNA needs to support efficient trans-translation.

\section{Conclusion}

This study significantly advances our understanding of trans-translation by demonstrating the existence of yet uncharacterized structural constraints in helices $2 \mathrm{~b}-2 \mathrm{~d}$, pk1 and the single-stranded sequence upstream of the ORF. Although these conserved segments of tmRNA are not believed to interact directly with $\mathrm{SmpB}$, they have a profound effect on the tagging activity of tmRNA:SmpB complexes. Moreover, our finding that tmRNA and SmpB can function in heterologous systems extends the investigation of trans-translation beyond the standard E. coli model and provides opportunities to target the trans-translaton system of $M$. tuberculosis for pharmacological intervention against multidrug-resistant tuberculosis $[11,47]$.

\section{Methods}

Bacterial strains and plasmids

E. coli strain XL1-B was the host for cloning. Expression strain IW764 was derived from E. coli BL21(DE3)/pLysS by deleting $s m p B$ and $s s r A$ genes. DNA fragment from BstXI restriction site in $s m p B$ and $S p h \mathrm{I}$ in $s s r A$ was replaced with the kanamycin-resistance gene from plasmid pACYC177. IW764 strain was constructed as described in [48].

Plasmids used in the study are listed in Table S1. Master plasmid pWOW was produced by modifying the pETrpmA-At-3 plasmid [24]. It has additional restriction sites at the start (NdeI) and at the end (EcoRI) of the $s m p B$ gene. These sites were used to replace E. coli $s m p B$ gene with $M$. tuberculosis $\operatorname{smp} B$ gene. Plasmid pWOW variants containing $M$. tuberculosis ssrA gene were constructed using 2-step PCR protocol described earlier [49]. DNA fragment encoding mature E. coli tmRNA was replaced with equivalent mature $M$. tuberculosis $s s r A$ gene. $M$. tuberculosis $s m p B$ and $s s r A$ genes remained under control of E.coli regulatory signals. E. coli and M. tuberculosis $s m p B$ genes were cloned between $N d e I$ and $X h o$ I restriction sites in protein expression vector $\mathrm{pET}$-23a. Overexpressed SmpB proteins have an additional LEHHHHHH tag at their C-termini.

\section{Purification of SmpB proteins}

E. coli and $M$. tuberculosis SmpB proteins were overexpressed in the IW764 strain. E. coli cells were incubated at $30^{\circ} \mathrm{C}$ and protein expression was induced in the mid-log phase following standard protocols. Proteins were extracted with the lysis buffer containing $100 \mathrm{mM}$ sodium phosphate, $10 \mathrm{mM}$ Tris, $8 \mathrm{M}$ urea ( $\mathrm{pH}$ 8.0) and purified on $\mathrm{Ni}^{2+}$-NTAagarose (Qiagen) under denaturing conditions and dialyzed against the storage buffer containing $50 \mathrm{mM}$ MES-KOH (pH 6.5), $200 \mathrm{mM} \mathrm{KCl,} 5 \mathrm{mM}$ 2-mercaptoethanol and 10\% glycerol. This procedure yields His-tagged SmpB proteins that are $>95 \%$ pure.

\section{Synthesis and purification of chimeric tmRNAs}

Plasmid ptmR with the ssrA gene derivatives under control of the T7 promoter was linearized with restriction enzyme BstNI and used as a template for the in vitro synthesis of chimeric tmRNAs as described earlier [10]. TmRNA transcripts were purified on RNeasy mini-spin columns according to instructions provided by the manufacturer (Qiagen). The integrity of the transcripts was tested by electrophoresis on a denaturing $5 \%$ polyacrylamide gel.

\section{In vivo tagging assay}

To monitor in vivo tagging of chimeric tmRNAs, we modified an earlier described procedure [24]. E. coli IW764 cells were transformed with plasmid pWOW coding for truncated protein L27 under control of T7 promoter and a combination of E. coli or M. tuberculosis SmpB and tmRNA variants under the control of native E.coli promoters. Cells were grown at $37^{\circ} \mathrm{C}$ in $2 \mathrm{xYT}$ broth supplemented with ampicillin $(200 \mathrm{mg} / \mathrm{mL})$, chloramphenicol $(30 \mathrm{mg} / \mathrm{mL})$ and 
kanamycin $(50 \mathrm{mg} / \mathrm{mL}) .1 \mathrm{mM}$ IPTG was added when $\mathrm{A}_{600}$ reached 0.3. After additional 3 hours of incubation, cells were collected by low-speed centrifugation. Cell pellets for RNA analysis were flash-frozen and stored at $-80^{\circ} \mathrm{C}$. Cells for protein analysis were lysed in a SDS-polyacrylamide gel-loading buffer at a concentration of $0.005 \mathrm{~A}_{600} / \mu \mathrm{L}$. $5-10 \mu \mathrm{L}$ aliquots of lysates were fractionated on a $12.5 \%$ SDS/Tricine-polyacrylamide gel and stained with Coomassie Brilliant Blue R-250 (Sigma).

\section{In vitro tagging assay}

All assays were performed with the E. coli T7 transcription/ translation system for circular DNA from Promega [22]. A typical $25-\mu \mathrm{L}$ reaction mixture contained $2 \mu \mathrm{g}$ of circular plasmid pETrpmA-At-1 encoding the gene for truncated ribosomal protein L27, 25 pmoles of His-tagged SmpB protein, 40 pmoles of tmRNA, and 20 units of SUPERase-In ${ }^{\text {TM }}$. Tagging reaction was carried out for $1 \mathrm{~h}$ at $37^{\circ} \mathrm{C}$. Tagged proteins were captured on Ni-NTA magnetic agarose beads (Qiagen), fractionated on a $10 \%$ SDS/Tricine-polyacrylamide gel and blotted to a Hybond-LFP membrane (GE Healthcare Life Sciences). Because His-tagged truncated protein L27 had T7-tag peptide on its $\mathrm{N}$-terminus, it could be detected using the anti-T7 tag monoclonal antibody from Invitrogen and the secondary Cy5-labeled antibody from Amersham. Band intensity was quantified using a Typhoon Phosphoimager and ImageQuant software (Amersham Biosciences).

\section{Detection of tmRNA in cell lysates}

Total bacterial RNA was extracted using RNeasy kit (Qiagen). Its concentration was measured using Quant-iT RNA assay kit from Invitrogen. Aliquots of 0.5 to $1 \mu \mathrm{g}$ of total RNA were fractionated on a denaturing 5\% polyacrylamide gel. RNA was blotted to a Zeta-probe membrane (Bio-Rad). Blots were probed with $\left[5^{\prime}-{ }^{32} \mathrm{P}\right]$ labeled oligonucleotides 5'-CAGCTGCGGACGGACAC3' and 5'-GTGAGTCCCTCTAGCTG-3' complementary to $E$. coli tmRNA(H8) and $M$. tuberculosis tmRNA(H6), respectively. Hybridization signals were visualized using a Typhoon 9410 Phosphoimager and ImageQuant software (GE Healthcare).

TmRNA:70S ribosome complexes were purified as described previously [19].

\section{Detection of protein SmpB in cell lysates}

Lysates of 0.02-0.03 $\mathrm{A}_{600}$ units of cells were fractionated on a $10 \%$ SDS/Tricine-polyacrylamide gel. Proteins were blotted to a Hybond-LFP membrane (GE Healthcare) by a wet transfer in Towbin buffer containing 20\% methanol and $0.05 \%$ SDS. Rabbit polyclonal antibodies raised against the E.coli SmpB protein and Cy5-labeled anti-rabbit secondary antibodies from ECL-Plex system (Amersham, GE Healthcare) were used to detect both E.coli and
M. tuberculosis SmpB proteins. Blots were visualized using Typhoon 9410 or LAS 4100 imagers and quantified using ImageQuant software (GE Healthcare).

SmpB proteins with the Tag-100 at their C-termini were detected with rabbit anti-Tag-100 monoclonal antibody from GenScript and anti-rabbit IgG-HRP using the ECL-Plus Western Blotting System (Amersham, GE Healthcare).

\section{Additional file}

Additional file 1: Table S1. Bacterial strains and plasmids. Table S2. Engineering chimeric tmRNAs. Figure S1. pWOW plasmid.

\section{Competing interests}

The authors declare that they have no competing interests.

\section{Authors' contributions}

IKW developed assays for testing the tagging activities of chimeric tmRNAs in the presence of either E. coli or M. tuberculosis SmpB proteins. CZ conceived the study and participated in all of its aspects. JW cloned, overexpressed and purified E. coli and M. tuberculosis SmpB proteins. All authors wrote and approved the manuscript.

\section{Acknowledgements}

The genomic DNA of M. tuberculosis H37RV was provided by Drs. Buka Samten and Xiuhau Pang at the University of Texas Health Northeast. This work was supported by the National Science Foundation [Grant 1063536] Funding for open access charge was provided by the Upchurch Fund for Excellence.

\section{Author details}

${ }^{1}$ Department of Animal Sciences, Auburn University, Auburn, AL 36849, USA ${ }^{2}$ Department of Biochemistry, University of Texas Health Science Center at San Antonio, San Antonio, TX 78229, USA.

Received: 27 March 2014 Accepted: 18 June 2014

Published: 15 September 2014

\section{References}

1. Moore SD, Sauer RT: The tmRNA system for translational surveillance and ribosome rescue. Annu Rev Biochem 2007, 76:101-124.

2. Keiler KC: Biology of trans-translation. Annu Rev Microbiol 2008, 62:133-151.

3. Felden B, Himeno H, Muto A, Atkins JF, Gesteland RF: Structural organization of Escherichia coli tmRNA. Biochimie 1996, 78:979-983.

4. Williams KP, Bartel DP: The tmRNA Website. Nucleic Acids Res 1998, 26:163-165.

5. Knudsen B, Wower J, Zwieb C, Gorodkin J: tmRDB (tmRNA database). Nucleic Acids Res 2001, 29:171-172.

6. Bessho Y, Shibata R, Sekine S, Murayama K, Higashijima K, Hori-Takemoto C, Shirouzu M, Kuramitsu S, Yokoyama S: Structural basis for functional mimicry of long-variable-arm tRNA by transfer-messenger RNA. Proc Natl Acad Sci USA 2007, 2007(104):8293-8298.

7. Rudinger-Thirion J, Giege R, Felden B: Aminoacylated tmRNA from Escherichia coli interacts with prokaryotic elongation factor Tu. RNA 1999, 5:989-992.

8. Barends S, Karzai AW, Sauer RT, Wower J, Kraal B: Simultaneous and functional binding of SmpB and EF-Tu-TP to the alanyl acceptor arm of tmRNA. J Mol Biol 2001, 314:9-21.

9. Wower IK, Jahan N, Zwieb C, Wower J: Ribosomal Protein S1: An Important Trans-Translation Factor. Biochemistry \& Physiology 2013, S2:1-7.

10. Wower IK, Zwieb CW, Guven SA, Wower J: Binding and cross-linking of tmRNA to ribosomal protein S1, on and off the Escherichia coli ribosome. EMBO J 2000, 19:6612-6621.

11. Shi W, Zhang X, Jiang X, Yuan H, Lee JS, Barry CE, Wang H, Zhang W, Zhang Y: Pyrazinamide inhibits trans-translation in Mycobacterium tuberculosis. Science 2011, 333:1630-1632. 
12. Saguy M, Gillet R, Skorski P, Hermann-Le Denmat S, Felden B: Ribosomal protein $\mathrm{S} 1$ influences trans-translation in vitro and in vivo. Nucleic Acids Res. 2007, 35:2368-2376.

13. Qi H, Shimizu Y, Ueda T: Ribosomal protein S1 is not essential for the trans-translation machinery. J. Mol. Biol. 2007, 368:845-852.

14. Takada K, Takemoto C, Kawazoe M, Konno T, Hanawa-Suetsugu K, Lee S, Shirouzu M, Yokoyama S, Muto A, Himeno H: In vitro trans-translation of Thermus thermophilus: ribosomal protein $\mathrm{S} 1$ is not required for the early stage of trans-translation. RNA 2007, 13:503-510.

15. Valle M, Gillet R, Kaur S, Henne A, Ramakrishnan V, Frank J: Visualizing tmRNA entry into a stalled ribosome. Science 2003, 300:127-130.

16. Kaur S, Gillet R, Li W, Gursky R, Frank J: Cryo-EM visualization of transfer messenger RNA with two SmpBs in a stalled ribosome. Proc Natl Acad Sci USA 2006, 103:16484-16489.

17. Cheng K, Ivanova N, Scheres SH, Pavlov MY, Carazo JM, Hebert H, Ehrenberg M, Lindahl M: tmRNA-SmpB complex mimics native aminoacyl-tRNAs in the A site of stalled ribosomes. J Struct Biol 2010, 169:342-348.

18. Fu J, Hashem Y, Wower I, Lei J, Liao HY, Zwieb C, Wower J, Frank J: Visualizing the transfer-messenger RNA as the ribosome resumes translation. EMBO J. 2010, 29:3819-3825.

19. Weis F, Bron P, Giudice E, Rolland JP, Thomas D, Felden B, Gillet R: tmRNA-SmpB: a journey to the centre of the bacterial ribosome. EMBO J 2010, 29:3810-3818.

20. Ramrath DJ, Yamamoto H, Rother K, Wittek D, Pech M, Mielke T, Loerke J, Scheerer $P$, Ivanov $P$, Teraoka $Y$, Shpanchenko O, Nierhaus KH, Spahn CM: The complex of tmRNA-SmpB and EF-G on translocating ribosomes. Nature 2012, 485:526-529.

21. Fu J, Hashem Y, Wower J: Frank J: tmRNA on its way through the ribosome. RNA Biology 2011, 8:586-590.

22. Wower IK, Zwieb C, Wower J: Escherichia coli tmRNA lacking pseudoknot 1 tags truncated proteins in vivo and in vitro. RNA 2009, 15:128-137.

23. Miller MR, Healey DW, Robison SG, Dewey JD, Buskirk AR: The role of upstream sequences in selecting the reading frame on tmRNA. BMC Biol 2008, 6:1-10.

24. Wower IK, Zwieb C, Wower J: Contributions of pseudoknots and protein $\mathrm{SmpB}$ to the structure and function of tmRNA in trans-translation. J Biol Chem 2004, 279:54202-54209.

25. Nameki N, Tadaki T, Himeno H, Muto A: Three of four pseudoknots in tmRNA are interchangeable and are substitutable with single-stranded RNAs. FEBS Lett 2000, 470:345-349.

26. Zucker M: Mfold web server for nucleic acid folding and hybridization. Nucleic Acids Res 2003, 31:3406-3415.

27. Mattice WL, Riser JM, Clark DS: Conformational properties of the complexes formed by proteins and sodium dodecyl sulfate. Biochemistry 1976, 15:4264-4272

28. Rath A, Glibowicka M, Nadeau VG, Chen G, Deber CM: Detergent binding explains anomalous SDS-PAGE migration of membrane proteins. Proc Natl Acad Sci USA 2009, 106:1760-1765

29. Tanner DR, Dewey JD, Miller MR, Buskirk AR: Genetic analysis of the structure and function of transfer messenger RNA pseudoknot 1. J Biol Chem 2006, 281:10561-10566.

30. Kapoor S, Samhita L, Varshney U: Functional significance of an evolutionarily conserved alanine (GCA) resume codon in tmRNA in Escherichia coli. J Bacteriol 2011, 193:3569-3576.

31. Wower J, Wower IK, Zwieb C: Making the jump: new insights into the mechanism of trans-translation. J Biol 2008, 7:1-4.

32. Gueneau de Novoa P, Williams KP: The tmRNA website: reductive evolution of tmRNA in plastids and other endosymbionts. Nucleic Acids Res 2004, 32:D104-D108.

33. Andersen ES, Rosenblad MA, Larsen N, Westergaard JC, Burks J, Wower IK, Wower J, Gorodkin J, Samuelsson T, Zwieb C: The tmRDB and SRPDB resources. Nucleic Acids Res 2006, 34:D163-D168.

34. Keiler KC, Shapiro L, Williams KP: tmRNAs that encode proteolysis-inducing tags are found in all known bacterial genomes: A two-piece tmRNA functions in Caulobacter. Proc Natl Acad Sci USA 2000, 97:7778-7783.

35. Williams KP: Descent of a split RNA. Nucleic Acids Res 2002, 30:2025-2030.

36. Zwieb C, Wower I, Wower J: Comparative sequence analysis of tmRNA. Nucleic Acids Res 1999, 27:2063-2071.

37. Roche ED, Sauer RT: SsrA-mediated peptide tagging caused by rare codons and tRNA scarcity. EMBO J 1999, 18:4579-4589.
38. Dong $H$, Nilsson L, Kurland CG: Co-variation of tRNA abundance and codon usage in Escherichia coli at different growth rates. J Mol Biol 1996, 260:649-663.

39. O'Connor M: Minimal translation of the tmRNA tag-coding region is required for ribosome release. Biochem Biophys Res Commun 2007, 357:276-281.

40. Thibonnier M, Aubert S, Ecobichon C, De Reuse H: Study of the functionality of the Helicobacter pylori trans-translation components SmpB and SsrA in an heterologous system. BMC Microbiol 2010, 10:1-11.

41. Williams KP, Martindale KA, Bartel DP: Resuming translation on tmRNA: a unique mode of determining a reading frame. EMBO J 1999, 18:5423-5433.

42. Lee S, Ishii M, Tadaki T, Muto A, Himeno H: Determinants on tmRNA for initiating efficient and precise trans-translation: some mutations upstream of the tag-encoding sequence of Escherichia coli tmRNA shift the initiation point of trans-translation in vitro. RNA 2001, 7:999-1012.

43. Konno T, Kurita D, Takada K, Muto A, Himeno $\mathrm{H}$ : A functional interaction of SmpB with tmRNA for determination of the resuming point of trans-translation. RNA 2007, 13:1723-1731.

44. Wower J, Zwieb CW, Hoffman DW, Wower IK: SmpB: a protein that binds to double-stranded segments in tmRNA and tRNA. Biochemistry 2002, 2002(41):8826-8836.

45. Gillet R, Kaur S, Li W, Hallier M, Felden B, Frank J: Scaffolding as an organizing principle in trans-translation. The roles of small protein $B$ and ribosomal protein S1. J Biol Chem 2007, 282:6356-6363.

46. Ivanov PV, Zvereva MI, Shpanchenko OV, Dontsova OA, Bogdanov AA, Aglyamova GV, Lim VI, Teraoka Y, Nierhaus KH: How does tmRNA move through the ribosome? FEBS Lett 2002, 514:55-59.

47. Personne $Y$, Parish T: Mycobacterium tuberculosis posseses an unusual tmRNA rescue system. Tuberculosis 2014, 94:34-42.

48. Wower IK, Wower J, Zimmermann RA: Ribosomal protein L27 participates in both $50 \mathrm{~S}$ subunit assembly and the peptidyl transferase reaction. J Biol Chem 1998, 273:19847-19852.

49. Chen GJ, Qiu N, Karrer C, Caspers P, Page MG: Restriction site-free insertion of PCR products directionally into vectors. Biotechniques 2000, 28:498-500. 504-505.

doi:10.1186/1471-2199-15-19

Cite this article as: Wower et al:: Requirements for resuming translation in chimeric transfer-messenger RNAs of Escherichia coli and Mycobacterium tuberculosis. BMC Molecular Biology 2014 15:19.

\section{Submit your next manuscript to BioMed Central and take full advantage of:}

- Convenient online submission

- Thorough peer review

- No space constraints or color figure charges

- Immediate publication on acceptance

- Inclusion in PubMed, CAS, Scopus and Google Scholar

- Research which is freely available for redistribution 